\title{
Capsule Commentary on Hess et al. A Randomized Controlled Pilot Trial of the Functional Assessment Screening Tablet to Engage Patients at the Point of Care
}

\author{
Erika Litvin Bloom, PhD \\ Department of Psychiatry and Human Behavior, Alpert Medical School of Brown University, Providence, RI, USA.
}

J Gen Intern Med 29(12): 1690

DOI: $10.1007 / \mathrm{s} 11606-014-3043-2$

(c) Society of General Internal Medicine 2014

$\mathrm{T}$ his pilot trial by Hess et al. ${ }^{1}$ compared patient and provider reports of discussions regarding health behaviors and mental and physical health-related quality of life (HRQoL) during a clinical encounter among patients who (1) received or (2) did not receive a health-information technology intervention that provided them with feedback prior to the encounter. The authors note that in previous research, reports of discussion of health behaviors by both patients and providers are infrequent ${ }^{2,3}$ and they speculate that lack of time ${ }^{4}$ (i.e., $<15 \mathrm{~min}$ appointments) contributes to fewer discussions.

Resident-physicians at an academic health center were randomized to have their patients either receive or not receive FAST-Feedback, a personalized information sheet about their health behaviors and HRQoL, after completing the FAST, an intake questionnaire delivered via a tablet computer. Results indicated no significant differences among patients who received vs. did not receive FASTFeedback in frequency of initiating any necessary discussion, the occurrence of any necessary discussion regardless of initiator, or the helpfulness of discussions. However, patients with low mental HRQoL who received FASTFeedback were more likely to report initiating discussions related to mental HRQoL. Also, resident-physicians whose patients received FAST-Feedback reported that more of their patients with low physical HRQoL initiated discussion of physical HRQoL. Agreement between patients and resident-physicians regarding whether discussions occurred and the helpfulness of these discussions was low.
This study had limitations, including a low sample size that limited power to detect group differences, a low participation rate ( $24 \%$ of eligible patients), and non-blinded randomization of resident-physicians rather than patients. The FASTFeedback intervention was also low in intensity, consisting of a single information sheet. Nevertheless, as the authors note, the finding that more discussions of mental health occurred in the intervention group is notable, as discussions about mental health may be especially challenging for both patients and physicians to initiate. Overall, this research is aimed at using newly available technologies to develop more efficient methods of providing primary care within the restrictions of the existing health care system.

Conflict of Interest: The authors declare that they do not have a conflict of interest.

Corresponding Author: Erika Litvin Bloom, PhD; Department of Psychiatry and Human Behavior, Alpert Medical School of Brown University, Providence, RI, USA (e-mail: Erika_bloom@brown.edu).

\section{REFERENCES}

1. Hess RH, Tindle H, Conroy MB, Clark S, Yablonsky E, Hays RD. A randomized controlled pilot trial of the Functional Assessment Screening Tablet to engage patients at the point of care. J Gen Intern Med. 2014. doi:10.1007/s11606-014-2984-9.

2. Glasgow RE, Eakin EG, Fisher EB, Bacak SJ, Brownson RC. Physician advice and support for physical activity: results from a national survey. Am $\mathrm{J}$ Prev Med. 2001;21(3):189-196.

3. AAMC. Physician Behavior and Practice Patterns Related to Smoking Cessation. Washington DC: AAMC; 2007.

4. Yarnall KS, Pollak KI, Ostbye T, Krause KM, Michener JL. Primary care: is there enough time for prevention? Am J Public Health. 2003;93(4):635641.

Published online September 30, 2014 